\title{
SE BUSCA GEOBIBLIOTECARIO: LOS DATOS GEOGRÁFICOS ENTRAN EN LA BIBLIOTECA
}

\section{Carlos Granell-Canut y Estefanía Aguilar-Moreno}
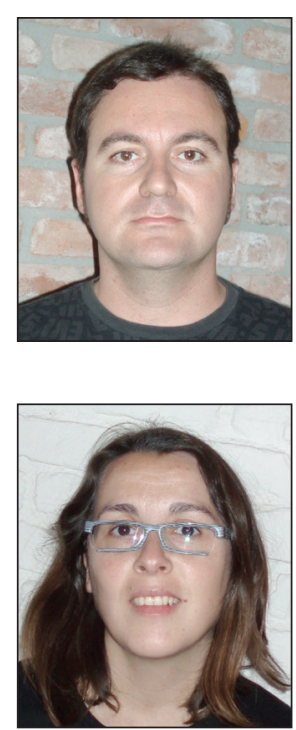

Carlos Granell-Canut, doctor en geoinformática por la Universitat Jaume I, es investigador postdoctoral en el Instituto de Medio Ambiente y Sostenibilidad del European Commission-Joint Research Centre ( $E C-J R C)$. Su investigación se centra en la aplicación de los sistemas de información geográfica y tecnologías geoespaciales y su conexión con otros tipos de datos, visto en conjunto como una gran infraestructura de datos, información y servicios a la disposición de los ciudadanos. http://orcid.org/0000-0003-1004-9695

European Commission-Joint Research Centre, Institute for Environment and Sustainability Via Enrico Fermi, 2749. 21027 Ispra, Italia carlos.granell@jrc.ec.europa.eu

Estefanía Aguilar-Moreno es licenciada en documentación y master en la sociedad de la información y el conocimiento por la Universitat Oberta de Catalunya (UOC). Ha trabajado durante más de 10 años en un centro de documentación especializado realizando, principalmente, tareas de vigilancia tecnológica e inteligencia competitiva (VT-IC). Investiga temas relacionados con la inteligencia estratégica y sus fronteras, e imparte formación. http://orcid.org/0000-0002-3157-2015

http://www.directorioexit.info/ficha1256 http://www.linkedin.com/in/estefaniaaguilar esamo66@gmail.com

\section{Resumen}

La mayor proliferación y accesibilidad de los datos geográficos están cambiando el panorama de servicios basados en información geográfica. A partir del análisis de recientes ofertas de trabajo en bibliotecas universitarias americanas se analiza cuál podría ser el rol del profesional de la información en este nuevo contexto, en cuanto a la gestión y difusión de las colecciones de datos geográficos, identificando sinergias para beneficio mutuo entre las bibliotecas universitarias con sus profesionales de la información y la comunidad que usa datos geográficos y sistemas de información geográfica (SIG). De hecho esta actividad sería una más, un caso particular, de las de curación de datos que se reclama para muchas disciplinas.

\section{Palabras clave}

Datos geográficos, Unidades de información, Gestión de colecciones, Servicios geográficos en bibliotecas, Geobibliotecario, Sistemas de información geográfica (SIG), Curación de datos.

\section{Title: Geo-librarian wanted: geographic data come to the library}

\section{Abstract}

The increased proliferation and accessibility of geographic data are changing the landscape of services based on geographic information. From the analysis of recent job offers posted in American university libraries, we discuss what could be the role of the information professional in this new context, with respect to the management and dissemination of geographic data collections, looking for synergies for mutual benefit between academic libraries and their information professionals and the geographic data community that uses geographic information systems (GIS). In fact, this activity would be one more among the data curation or data sets preservation tasks needed currently in many disciplines.

\section{Keywords}

Geographic data, Information units, Collection management, Geographic services in libraries, Geolibrarian, Geographic information systems (GIS), Data curation, Data sets.

Granell-Canut, Carlos; Aguilar-Moreno, Estefanía. "Se busca geobibliotecario: los datos geográficos entran en la biblioteca". El profesional de la información, noviembre-diciembre, v. 22, n. 6, pp. 569-575. 


\section{Datos geográficos: nueva colección a gestionar}

El panorama bibliotecario español está cambiando. Así lo demuestra el informe de 2012 del Panorama de las $52 \mathrm{Bi}$ bliotecas Públicas de Estado ${ }^{1}$. Su cambio forzoso viene dado por varias circunstancias: recortes presupuestarios, nuevas demandas por parte de los usuarios, y avances tecnológicos. Las consecuencias de los recortes presupuestarios son ya visibles en el cierre de bibliotecas y la reducción en personal. La época de cambios, marcada por el contexto económico de los últimos años, se ve agravada por las nuevas conductas sociales y la adopción masiva de nuevas tecnologías de la comunicación -libros electrónicos, dispositivos móviles y redes sociales- que obligan a las bibliotecas a actualizarse, puesto que los usuarios demandan nuevas formas de ponerse en contacto (Carrión-Gútiez, 2013) y hacer uso de sus servicios. Las bibliotecas se esfuerzan por adaptarse, ya que estas tecnologías aun siendo en principio externas a las funciones propias de la biblioteca, se han convertido en herramientas necesarias para su funcionamiento debido a su omnipresencia en la sociedad.

Frente a la estrategia de importación de métodos y tecnología externos para estar en concordancia con los cambios sociales, las bibliotecas también pueden potenciar y proyectar sus capacidades hacia el exterior, es decir, exportando y aplicando los conocimientos y el saber hacer bibliotecario a otros usos y contextos. Uno de los puntos fuertes, por no decir el pilar fundamental de las bibliotecas y sus profesionales, es la gestión y mantenimiento de las colecciones. Sean estas colecciones del tipo que sean, físicas o digitales, el profesional de la información ha venido organizándolas, indexándolas y preservándolas desde su creación. En este artículo vamos a particularizar en las colecciones de datos geográficos digitales.

En las ofertas de empleo se solicitaba formación en información y documentación, complementada con conocimientos de SIG

Los datos geográficos en formato digital no son únicamente el resultado de las digitalización de cartografía histórica, cartas de navegación y otros tipos de mapas creados originalmente en formato papel (Badia et al., 2005; Solar; Radovan, 2005; Edelson; Ferster, 2013). A diferencia de los mapas antiguos, casi artísticos pero estáticos, los mapas digitales actuales son productos generados dinámicamente como resultado de la superposición de diversas capas de información a gusto del usuario. Cada una de estas capas es un conjunto de datos geográficos, es decir, datos que llevan asociada una posición geográfica que, al estar conectados con otros datos y puestos sobre un mapa base, dan a la información presentada mayor sentido (Aguilar-Moreno; Granell-Canut, 2013). La combinación de diferentes capas de información ofrece una nítida representación espacial y por lo tanto posibilita su uso como herramienta de visualización de la información y toma de decisiones.
La pregunta que analizamos es si, por las características que presentan los datos geográficos, estas colecciones podrían ser gestionadas por bibliotecas universitarias y/o por centros de investigación, ampliando de esta forma el abanico de servicios ofrecidos por las bibliotecas. De hecho se trataría de una actividad de curación de datos (data curation) o de preservación de conjuntos de datos (data sets) para que se puedan reutilizar, una tendencia de la que se habla cada vez con más intensidad desde hace unos pocos años. Algunas agencias de financiación de la investigación ya exigen que en las solicitudes de subvención de proyectos figure un plan de depósito de los datos utilizados en vistas a su reutilización por otros investigadores.

\section{Hacia un nuevo rol: geobibliotecario}

En el período de un mes (de finales de mayo de 2013 a finales de junio de 2013) ALA Joblist publicó cinco ofertas de trabajo que relacionaban los datos geográficos con el mundo de las bibliotecas. Todas estaban radicadas en bibliotecas universitarias estadounidenses y esto nos llevó a pensar que podía ser interesante estudiar las competencias requeridas para este perfil tan específico.

La tabla 1 muestra un análisis de las ofertas de trabajo aparecidas $^{2}$, recogiendo los requisitos que el demandante de empleo debía cumplir así como las principales funciones y competencias requeridas para estos puestos de trabajo. Las " $X$ " marcan competencias exigidas para el puesto y los "?" señalan que esa competencia se indica como preferible, pero no necesaria.

Si quitásemos los términos SIG (sistemas de información geográfica) o geográfico a la lista de funciones de la tabla 1, vemos que cualquiera de las tareas aquí enumeradas son exactamente aquellas que el profesional de la información desempeña habitualmente. Todas las ofertas de empleo destacan que, como requisito fundamental, el candidato/a tenga diploma o master en documentación complementado con conocimientos en SIG. Además, en todas ellas se hace hincapié, como tareas preferibles a desempeñar, el mantenimiento de la colección de datos geográficos (Florance, 2007) que incluye la organización, análisis y preservación (Sweetkind-Singer et al., 2006), así como la gestión de metadatos, ofrecer servicios de referencia y difusión, y llevar a cabo actividades de enlace entre departamentos que lleven a cabo actividades relacionadas con SIG (Weimer; Reehling, 2006).

Quizá lo más importante de las ofertas de empleo anteriores es que se pide como formación primaria información y documentación, complementada con conocimientos de SIG a nivel de experiencia y/o formación. En este sentido, se conserva la figura del documentalista/bibliotecario como formación primaria pero con especialización en SIG.

Es una cuestión de adaptarse o morir, tanto para el bibliotecario, como para la biblioteca. Los servicios que ofrecen las bibliotecas universitarias deben adaptarse a los cambios sociales y tendencias tecnológicas (Anglada, 2012). La realidad es que la información geográfica es un tipo de datos omnipresente en la vida cotidiana, y los campus universitarios no escapan a esta tendencia. Es más, los centros de conocimiento (universidades, centros de investigación...), junto con la administración pública, se encuentran entre las 
Tabla 1. Sistematización de las ofertas de trabajo para bibliotecarios en sistemas de información geográfica

\begin{tabular}{|c|c|c|c|c|c|}
\hline & $\begin{array}{l}\text { NY University } \\
\text { Libraries } \\
(27 / 06 / 2013)\end{array}$ & $\begin{array}{c}\text { Pennsylvania State } \\
\text { University Libraries } \\
(24 / 06 / 2013)\end{array}$ & $\begin{array}{c}\text { Lafayette } \\
\text { College } \\
(17 / 06 / 2013) \\
\end{array}$ & $\begin{array}{c}\text { University of } \\
\text { California, Berkeley } \\
(30 / 05 / 2013)\end{array}$ & $\begin{array}{c}\text { Ohio State Uni- } \\
\text { versity Libraries } \\
(30 / 05 / 2013)\end{array}$ \\
\hline \multicolumn{6}{|l|}{ Requisitos } \\
\hline Diploma o master en bibliotecas & $x$ & $x$ & & $x$ & $x$ \\
\hline Conocimientos prácticos SIG & $x$ & $x$ & $x$ & $x$ & $x$ \\
\hline Formación específica en SIG & $?$ & $x$ & & $?$ & \\
\hline \multicolumn{6}{|l|}{ Funciones y competencias } \\
\hline Servicio de referencia & $\mathrm{X}$ & $X$ & & $\mathrm{X}$ & $\mathrm{X}$ \\
\hline $\begin{array}{l}\text { Actividades de enlace entre departa- } \\
\text { mentos que lleven a cabo actividades } \\
\text { haciendo uso de SIG }\end{array}$ & $x$ & $x$ & $x$ & $x$ & $x$ \\
\hline $\begin{array}{l}\text { Participación en grupos de investiga- } \\
\text { ción internos sobre SIG }\end{array}$ & $x$ & $\mathrm{X}$ & & & \\
\hline $\begin{array}{l}\text { Desarrollo de proyectos e iniciativas } \\
\text { en SIG }\end{array}$ & & $\mathrm{X}$ & & $x$ & \\
\hline Alfabetización en SIG & $x$ & $x$ & & $x$ & \\
\hline $\begin{array}{l}\text { Mantenimiento de la colección: } \\
\text { creación, organización, análisis y } \\
\text { preservación }\end{array}$ & $x$ & $x$ & & $x$ & $x$ \\
\hline Metadatos geográficos & $x$ & & & $x$ & \\
\hline $\begin{array}{l}\text { Identificación de necesidades en datos } \\
\text { SIG }\end{array}$ & & $\mathrm{X}$ & & & \\
\hline Actividades de difusión & & $x$ & $x$ & $\mathrm{X}$ & $x$ \\
\hline $\begin{array}{l}\text { Promoción de habilidades en visualiza- } \\
\text { ción de datos geográficos }\end{array}$ & & & $x$ & & $x$ \\
\hline $\begin{array}{l}\text { Elaboración de recursos de informa- } \\
\text { ción sobre SIG }\end{array}$ & & & & $x$ & \\
\hline $\begin{array}{l}\text { Participación en eventos como repre- } \\
\text { sentantes SIG del centro }\end{array}$ & & & & & $X$ \\
\hline
\end{tabular}

organizaciones que más datos geográficos pueden generar y, al tiempo, necesitar para llevar a cabo proyectos de investigación (Longley et al., 2010). Es un hecho, que la compartición de datos de investigación en general y de datos geográficos en particular, como un tipo de datos especializado, está tomando cada vez más relevancia, puesto que las políticas de investigación europeas para futuros proyectos de investigación requerirán planes de gestión de los datos científicos generados de la propia investigación (Torres-Salinas et al., 2012; González et al., 2013).

\section{El flujo de trabajo y la metodología de} gestión de las colecciones de datos geográficos son similares al saber hacer de un bibliotecario

Otras funciones interesantes, aunque no identificadas en todas las ofertas como tareas prioritarias, son la participación en grupos de investigación internos sobre SIG, desarrollo de proyectos e iniciativas en SIG, y la alfabetización en SIG ( $\mathbf{N i -}$ cholson, 2007). Es decir, una parte de las competencias se centra en interaccionar y colaborar con otros miembros de la comunidad universitaria interesados en datos geográficos desde diferentes puntos de vista: como creador o como usuario de datos geográficos.
Las ofertas anteriores están acotadas al contexto de las bibliotecas universitarias americanas, lo cual no significa que esta tendencia deba reflejarse con la misma intensidad en el panorama español ${ }^{3}$. Sin embargo no hemos localizado ni ofertas ni puestos de este tipo en el contexto español y nos preguntamos el porqué de esta situación si, como hemos visto anteriormente, no resultaría un gran obstáculo la gestión de colecciones de datos geográficos por las bibliotecas. De entre las razones que nos vienen a la mente, resaltamos el desconocimiento mutuo aparente entre los profesionales de la información y la comunidad SIG (Boxall, 2002; López-Morales, 2008). Desde la perspectiva bibliotecaria, por ejemplo, el desconocimiento de la existencia de datos geográficos como colección a gestionar junto con la percepción de que SIG se relaciona más con un perfil informático que con uno bibliotecario, pueden ser motivos de falta de colaboración. Por otro lado, desde la perspectiva SIG, el desconocimiento de las tareas habituales de un bibliotecario en cuanto a la gestión de colecciones, puede evidenciar la falta de acercamiento entre estas dos comunidades.

De hecho, el perfil de "geobibliotecario" ni existe explícitamente ni parece que se perciba aún como necesario en el contexto español. No obstante, estas ofertas están marcando una clara tendencia en cuanto a las nuevas necesidades y roles que las bibliotecas pueden y deben adoptar para mantener su protagonismo en la sociedad en general y en el contexto universitario en particular (Vardakosta; Kapidakis, 2013). 


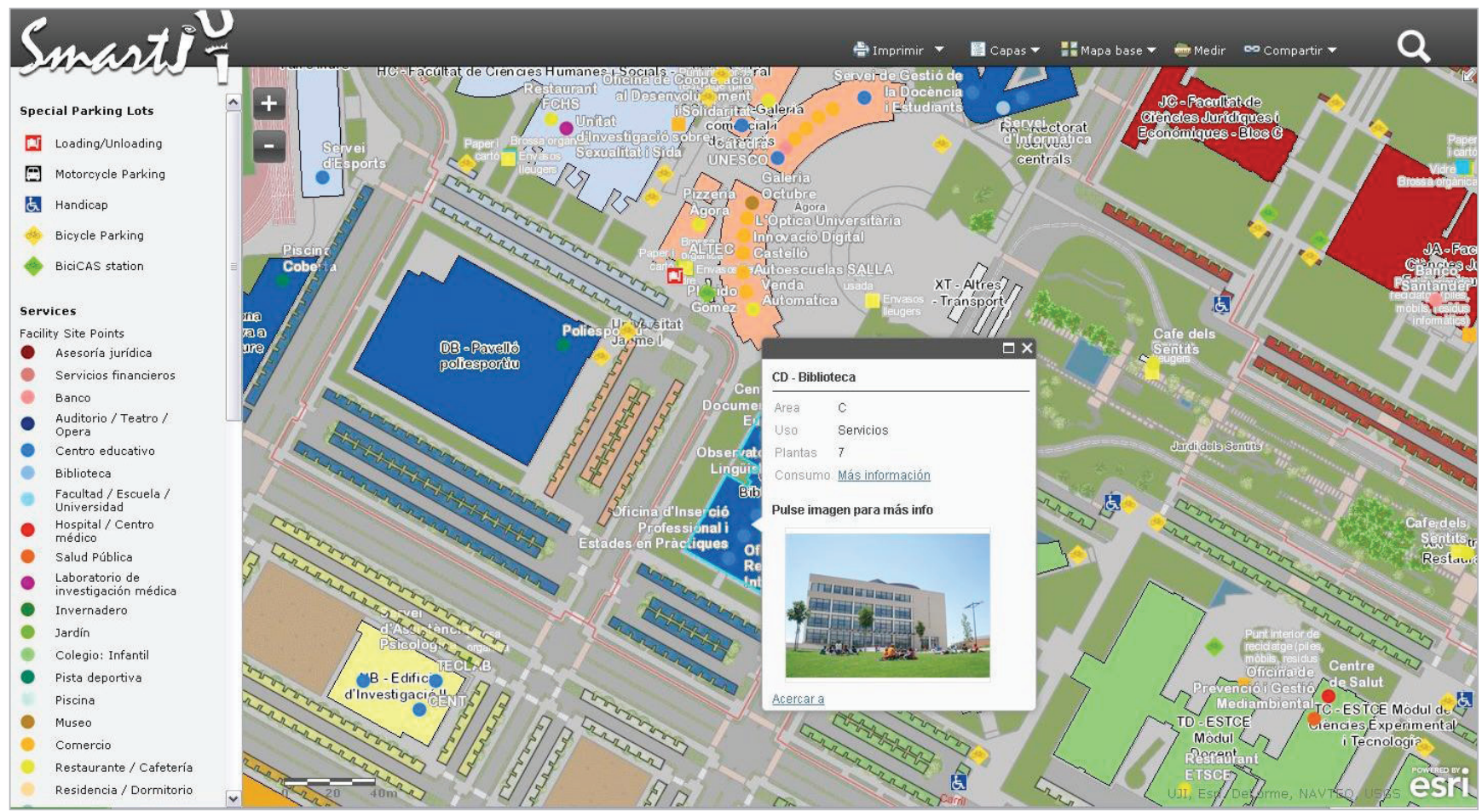

Figura 1. SmartUJI: Aplicación Smart campus de la Universitat Jaume I de Castellón

http://smart.uji.es/smartweb/index.php

En las siguientes dos secciones destacamos las sinergias entre bibliotecas y datos geográficos e identificamos nuevas oportunidades que están al alcance de los geobibliotecarios en el contexto de las bibliotecas universitarias, como ejemplos ilustrativos de las posibilidades que ofrece el binomio documentación y SIG.

Los datos geográficos dejan de ser algo específico de unos pocos para convertirse en un servicio troncal para la comunidad universitaria

\section{Las universidades respiran datos geográficos}

No es obvio pensar que las tareas de una biblioteca y de SIG tienen en común más semejanzas que diferencias. Sin embargo, si analizamos cuál es la esencia de las tareas específicas en cuanto a gestión de colecciones, en ambos casos se crean recursos, se documentan (metadatos) utilizando estándares, se registran los metadatos en catálogos públicos y se utilizan estándares y protocolos de comunicación abiertos para el acceso y descubrimiento de estos recursos en la Web. Visto desde esta perspectiva, el flujo de trabajo y la metodología de gestión de las colecciones de datos geográficos son bastante similares al saber hacer de un bibliotecario.

A priori, entonces, cualquier bibliotecario o documentalista podría gestionar colecciones de datos geográficos, puesto que partiendo de unos conocimientos en información y documentación de base, se necesitarían únicamente ciertas competencias relativas a los SIG para abarcar este nuevo nicho. No debería ser difícil llevar a cabo formación de personal con conocimientos en ambos campos, al igual que existen unidades de información especializadas en la gestión de colecciones específicas como sucede, por ejemplo, en centros de documentación de hospitales. Otra cosa, que escapa a este artículo, es verificar la existencia de cursos de formación especializados en documentación y SIG en España, aunque ya hay experiencias en EUA (Folse; Been, 2004; Weimer; Reehling, 2006; Miller, 2010).

A estas alturas, nadie pone en duda que las bibliotecas universitarias son un servicio central para las universidades, y como tales, proporcionan servicios troncales a toda la comunidad universitaria. De forma implícita, las ofertas de la tabla 1 ponen de relieve la naturaleza transversal de las colecciones de datos geográficos para distintas disciplinas y departamentos universitarios. Los datos geográficos dejan de ser algo específico de unos pocos para convertirse en un servicio troncal para la comunidad universitaria. Este es un aspecto importante que ya han identificado algunas universidades americanas pero que parece no percibirse en el contexto español.

Sin embargo, puede que la universidad española no esté tan lejos como cree de necesitar la figura del geobibliotecario. Como ejemplo ilustrativo, tan sólo hay que fijarse en la proliferación de los proyectos Smart campus (figura 1), o campus inteligente, promovidos por las universidades españolas con el fin de reducir consumos energéticos y mejorar la eficiencia en uso de recursos de los propios campus. En este tipo de proyectos es imprescindible la adecuada gestión, creación, acceso, compartición y provisión de los datos geográficos generados y relativos al campus. Las colecciones de datos de las infraestructuras, edificios, mobiliario del campus, inventarios de los árboles, y calles, así como también la información creada por los propios estudiantes, personal de la universidad y visitantes como resultados de geoposicio- 
nar los datos generados por éstos (Been, 2004). Como resultado, algunas de estas colecciones de datos se elevan a nivel de datos de "referencia", entendidos como los que sirven de base para la creación de otras tantas aplicaciones (buscadores de edificios o despachos como en la figura 1), o incluso para la generación de datos "temáticos", entendidos como agregaciones de capas de datos geográficos que, en conjunto, son un producto final para el usuario como por ejemplo mapas de movilidad dentro del campus, mapas de consumo energético, de ruido o de calidad del aire. Claramente, la oportunidad para los geobibliotecarios ya está presente debido a la acuciante necesidad de los campus universitarios en promocionar iniciativas inteligentes para el consumo y desarrollo sostenible. Por lo tanto, la gestión de colecciones de datos horizontales como los datos geográficos para proyectos e iniciativas vertebrales en las universidades debe ser el nuevo nicho de los geobibliotecarios.

Según las ofertas analizadas, el geobibliotecario no actúa solo, sino como parte de un grupo de expertos multidisciplinar relacionado con SIG y gestión de colecciones. Se prima mucho la colaboración, interacción, y el establecimiento de grupos multidisciplinares en torno a las necesidades de gestión de los datos geográficos (Dixon, 2006). Por ejemplo, personal técnico y expertos en la materia de la propia universidad pueden recomendar herramientas software SIG, especificaciones y formatos de datos para el apoyo de los nuevos servicios de la biblioteca. Miller (2010) aborda esta cuestión y enumera algunos proyectos de investigación en los que los geobibliotecarios han formado parte de un equipo de trabajo multidisciplinar.

La gestión de colecciones de datos horizontales como los datos geográficos para proyectos e iniciativas vertebrales en las universidades debe ser el nuevo nicho de los geobibliotecarios

Cabe destacar que es difícil encontrar un departamento con el nombre SIG en las universidades españolas, cosa que no ocurre en otras universidades europeas y del resto del mundo. A nivel de grupo de investigación ya es más fácil toparse con grupos dedicados a SIG y a las tecnologías y datos geográficos. Sin embargo, vale la pena tener en cuenta que otros muchos grupos y departamentos son, o bien usuarios de herramientas SIG y consumidores de datos geográficos, o bien productores de datos geográficos, aunque no se indique explícitamente. Algunos ejemplos pueden ser las facultades o departamentos de ciencias experimentales, donde grupos de hidrología o biología generan datos geográficos en sus experimentos y estudios. O pertenecientes a las ciencias sociales, donde los investigadores se nutren de datos geográficos para estudiar los patrones de movilidad urbana trazados sobre un mapa. Por lo tanto encontramos, en la misma universidad, diversos perfiles y disciplinas que utilizan tecnologías SIG y datos geográficos desde diferentes perspectivas. Grupos de trabajo mixtos que involucren a estos actores en colaboración con geobibliotecarios, podrían sentar las bases de un servicio de referencia geográfico. La gestión, mantenimiento y preservación de colecciones de datos geográficos y servicios relacionados podrían ser útiles para un sinfín de proyectos en marcha y en el futuro (Miller, 2010).

Grupos de trabajo mixtos, que aunen investigadores y geobibliotecarios, podrían sentar las bases de un servicio de referencia geográfico

\section{Comunidad SIG y bibliotecarios: obligados a entenderse}

Las ofertas de la tabla 1 enfatizan una tarea primordial de los bibliotecarios: gestionar las colecciones de datos geográficos. No es nuevo que las bibliotecas gestionen colecciones de recursos para la comunidad universitaria como sucede, por ejemplo, con los repositorios institucionales de artículos científicos. En este caso, el personal de la biblioteca solicita a los investigadores los artículos que han publicado y se encarga de generar los metadatos correspondientes, publicar todo junto en el repositorio, y suministrar al investigador el handler para que tenga acceso al artículo de forma permanente (Keefer, 2007). El investigador produce el recurso (en forma de artículo científico) y el bibliotecario se encarga de su ciclo de vida creando los metadatos, y cuidando del mantenimiento, documentación y registro para facilitar el acceso público y compartición.

Existe un paralelismo entre los repositorios y los datos geográficos: necesitamos que alguien se haga cargo de su gestión. En el caso de los repositorios se ha encontrado el camino, pero la comunidad SIG aún se encuentra ante este problema. ¿Quién debe documentar los datos geográficos que se van generando continuamente? ¿El creador del recurso? Así es en algunos casos. ¿Un especialista en documentación? Debería, pero no es la práctica habitual. Parece que hay un gran vacío cuando se aborda la creación de metadatos de datos geográficos. En general, se percibe como un aspecto vital para la comunidad SIG, ya que es una tarea fundamental en el ciclo de vida de un recurso, pero no se identifica claramente quién o qué perfil puede desempeñar ese rol. Evidentemente, existen herramientas software de generación de metadatos que hasta cierto punto ayudan a rellenar algunos campos de los registros, pero la supervisión humana experta es aún necesaria. Tal y como ocurre con el ejemplo del mantenimiento de la colección de artículos científicos en un repositorio institucional, ¿podría ser el bibliotecario la persona adecuada por formación y conocimientos para la catalogación y documentación de las colecciones de datos geográficos? Parece una buena idea que los geobibliotecarios participaran activamente en la gestión de las colecciones de datos geográficos, pues es la gestión de colecciones es la tarea natural y por excelencia de las bibliotecas universitarias. A su vez, se involucraría a la comunidad SIG y las bibliotecas universitarias en proyectos comunes. 
Sucede lo mismo que con los repositorios institucionales, donde se solicita la colaboración a los autores para que archiven sus trabajos en los repositorios, pero luego es necesaria la participación activa de los bibliotecarios para documentar, revisar errores y homogeneizar las colecciones de artículos científicos. Los geobibliotecarios, como parte del equipo multidisciplinar dentro de la universidad, darían sentido y unicidad a la colección de datos geográficos para que ésta fuera fácilmente accesible para la comunidad universitaria (Anglada, 2012).

Los datos geográficos destacan por su transversalidad y diversidad de usos y aplicaciones, y es por esto que la biblioteca -abierta y universal- ocupa una posición privilegiada para servir de nexo entre usuarios

\section{Conclusiones}

Los datos geográficos no son una moda pasajera, vienen para quedarse. Partiendo de la visión de la biblioteca universitaria como centro gestor de colecciones de datos que nos da Anglada (2012), este trabajo ahonda en un tipo concreto de colección, los datos geográficos, como parte del universo de información en el que las bibliotecas universitarias y centros de información deben involucrarse y participar activamente. La involucración de la biblioteca-crai en la curación de los conjuntos de datos que han servido para realizar investigaciones es una tendencia que va a más, aunque todavía existan dudas metodológicas sobre cuál es la mejor forma de preservarlos (Nina-Alcocer; Blasco-Gil; Fernanda Peset, 2013).

Los datos geográficos presentan un vacío en cuanto a quién lleva a cabo o supervisa la documentación y los metadatos. Los profesionales que trabajan con SIGs no sienten que sean ellos quienes deban documentar los datos que generan o actualizan. En la mayoría de ocasiones se desconoce que es el profesional de la información quien puede apoyar en las tareas de gestión de colecciones, tan necesarias en tantas disciplinas. Por otra parte se considera que la especificidad de los datos geográficos pueden no hacer viable la participación de los bibliotecarios, por falta de conocimientos técnicos, pero es ahí justo donde entra la formación y la capacidad de los profesionales de la información de situarse en el límite de ambas disciplinas.

Como se ha explicado en el primer punto, los datos geográficos destacan por su transversalidad y diversidad de usos y aplicaciones, y es por esto que la biblioteca -abierta y universal- ocupa una posición privilegiada para servir de nexo entre usuarios con diferentes perfiles y necesidades. Los hábitos de consumo de datos geográficos han cambiado y sus usuarios son cada vez más hábiles en su manejo, integración y combinación (Perera, 2008), por lo que esperan encontrar una ayuda experta por parte de los geobibliotecarios.

La participación de los bibliotecarios en proyectos SIG, como podemos suponer, no es directa, es necesaria for- mación y práctica en el tratamiento de estos datos. No sólo para saber gestionarlos, sino para usarlos y aplicarlos a nivel de usuario, y servir de referencia a potenciales usuarios (Holley, 2003). Como profesionales de la información, debemos ser conscientes de que, una vez finalizados nuestros estudios, es bien probable que necesitemos una especialización en algún campo en concreto. Como se presenta en este artículo, creemos que los datos geográficos y SIG no sólo aparecen como una forma de reciclaje y ampliación del horizonte profesional, sino como una oportunidad para mantener vivos los servicios de la biblioteca, siendo útiles a las nuevas necesidades de la comunidad universitaria y, por extensión, a la sociedad.

\section{Notas}

\section{1. http://www.mcu.es/bibliotecas/MC/EBPE/index.html}

2. Enlaces a las ofertas de trabajo analizadas:

- The Ohio State University Libraries. Geospatial information librarian. Publicada el 30 de mayo de 2013. http://joblist.ala.org/modules/jobseeker/GeospatialInformation-Librarian/23080.cfm

- University of California, Berkeley. GIS \& map librarian. Publicada el 30 de mayo de 2013.

http://joblist.ala.org/modules/jobseeker/GIS--MapLibrarian/23076.cfm

- Lafayette College, Skillman Library, Pennsylvania. Geospatial information systems librarian/specialist. Publicada el 17 de junio de 2013.

http://joblist.ala.org/modules/jobseeker/GeospatialInformation-Systems-LibrarianSpecialist--SkillmanLibrary/23291.cfm

- The Pennsylvania State University Libraries. Geospatial services librarian. Publicada el 24 de junio de 2013. http://www.libraries.psu.edu/psul/jobs/facjobs/GEOL. html

- New York University Libraries. Librarian for geospatial information systems/services. Publicada el 27 de junio de 2013.

http://joblist.ala.org/modules/jobseeker/Librarian-forGeospatial-Information-System-GIS-Services/23439.cfm

3. Desde una perspectiva no bibliotecaria, dentro del ámbito hispano, encontramos la iniciativa piloto Geobiblioteca, promovida por el Departamento de Geografía de la Facultad de Ciencias Humanas de la Universidad Nacional de Colombia, para la administración y distribución de datos espaciales. http://www.humanas.unal.edu.co/geobiblioteca/index.php

\section{Bibliografía}

Aguilar-Moreno, Estefanía; Granell-Canut, Carlos (2013). "Descubriendo los sistemas de información geográfica (SIG) para unidades de información". El profesional de la información, v. 22, n. 1, pp. 80-86.

http://dx.doi.org/10.3145/epi.2013.ene.11

Anglada, Lluís (2012). "Bibliotecas universitarias: cabalgando la tecnología, siguiendo al usuario". El profesional de la información, v. 21, n. 6, pp. 553-556. 
http://dx.doi.org/10.3145/epi.2012.nov.01

Badia, Ester; Carnerero, Francesc; González, Alfredo; Ordóñez, Rosalía (2005). "E-portal de cartografía de las bibliotecas de la UPC: acceso a las colecciones mediante un mapa sensible". EI profesional de la información, v. 14, n. 6, pp. 469-447. http://dx.doi.org/10.3145/epi.2005.nov.10

Been, Joshua (2004). "Broadening the use of GIS on campus: ArcIMS as e-reserves". En: ESRI education use conf procs. http://gis.esri.com/library/userconf/educ04/papers/ abstracts/a5148.html

Boxall, James (2002). "Geolibraries, the global spatial data infrastructure and digital Earth: A time for map librarians to reflect upon the Moonshot". Journal of the International Federation of Library Associations, v. 36, n. 1, pp. 1-21. http://www.ifla.org/IV/ifla67/papers/130-166e.pdf

Carrión-Gútiez, Alejandro (2013). "Informe de situación de las bibliotecas públicas españolas en 2012". El profesional de la información, v. 22, n. 3, pp. 250-258. http://dx.doi.org/10.3145/epi.2013.may.09

Dixon, Janet B. (2006). “Essential collaboration: GIS and the academic library". Journal of map \& geography libraries, v. 2, n. 2, pp. 5-20.

http://dx.doi.org/10.1300/J230v02n02_02

Edelson, S. Max; Ferster, Bill (2013). "MapScholar: a web tool for publishing interactive cartographic collections". Journal of map \& geography libraries, v. 9, n. 1-2, pp. 81-107 http://dx.doi.org/10.1080/15420353.2012.747463

Florance, Patrick (2006). "GIS collection development within an academic library". Library trends, v. 55, n. 2, pp. 222-235. http://dx.doi.org/10.1353/lib.2006.0057

Folse, Kimberly A.; Been, Joshua (2004). "Faculty and their institutional librarians: Developing labor capital by using GIS to teach social science". Journal of the National Social Science Association, v. 22, n. 1, pp. 21-27.

González, Luis-Millán; Saorín, Tomás; Ferrer-Sapena, Antonia; Aleixandre-Benavent, Rafael; Peset, Fernanda (2013). "Gestión de datos de investigación: infraestructuras para su difusión". El profesional de la información, v. 22, n. 5, pp. 415-423. http://dx.doi.org/10.3145/epi.2013.sep.06

Holley, Rose (2003). "The new map librarians: local perspective and local experience on digital spatial data and map librarianship". En: GeoCart 2003.

http://eprints.rclis.org/7486

Keefer, Alice (2007) "Los repositorios digitales universitarios y los autores". Anales de documentación, v. 10, pp. 205-214. http://revistas.um.es/analesdoc/article/view/1151/1201

Longley, Paul A.; Goodchild, Michael F.; Maguire, David J.; Rhind, David W. (2011). Geographic information sys- tems and science. Hoboken: John Willey \& Sons. ISBN: 978 0470721445

López-Morales, Carmen-Yasmina (2008). “La integración de sistemas de información geográfica. Parte de los servicios de la Biblioteca Daniel Cosío Villegas del Colegio de México". Reencuentro, n. 51, marzo, pp. 83-89.

Miller, Chris C. (2010). "Library applications, collaborations and courses for geodata and geoinformatics". En: $31^{\text {st }} \mathrm{An-}$ nual conf of the International Association of Scientific and Technical University Libraries, paper 6. http://docs.lib.purdue.edu/iatul2010/conf/day2/6

Nicholson, Andrew (2007). "Spatial literacy and information literacy: An evolution of GIS services in libraries". Bulletin of the Association of Canadian Map Libraries and Archives, $\mathrm{n}$. 129, pp. 3-5.

http://collections.mun.ca/cdm4/document. php?CISOROOT $=/$ acmla $\& C I S O P T R=6137 \& R E C=7$

Nina-Alcocer, Víctor; Blasco-Gil, Yolanda; Peset, Fernanda (2013). "Datasharing: guía práctica para compartir datos de investigación". El profesional de la información, nov.-dic., v. 22, n. 6, pp. 554-561.

http://dx.doi.org/10.3145/epi.2013.nov.?09

Perera, Pushpamala (2008). "The (unknown) role of map librarian and the challenges faced in satisfying the cartographic user needs". En: National conf on library \& information studies (Naclis 2008).

http://eprints.rclis.org/12137

Solar, Renata; Radovan, Dalibor (2005). "Use of GIS for presentation of the map and pictorial collection of the $\mathrm{Na}$ tional and University Library of Slovenia". Information technology \& libraries, v. 24, n. 4, pp. 196-200.

Sweetkind-Singer, Julie; Larsgaard, Mary-Lynette; Erwin, Tracey (2006). "Digital preservation of geospatial data". Library trends, v. 55, n. 2, pp. 304-314.

http://dx.doi.org/10.1353/lib.2006.0065

Torres-Salinas, Daniel; Robinson-García, Nicolás; CabezasClavijo, Álvaro (2012). "Compartir los datos de investigación en ciencia: introducción al data sharing". El profesional de la información, v. 21, n. 2, pp. 173-184.

http://dx.doi.org/10.3145/epi.2012.mar.08

Vardakosta, Ifigenia; Kapidakis, Sarantos (2013). "The new trends for librarians in management of geographic information". Procedia - Social and behavioral sciences, v. 73, pp. 794-801.

Weimer, Katherine H.; Reehling, Pete (2006). "A new model of geographic information librarianship: Description, curriculum and program proposal". Journal of education for library \& information science, v. 47, n. 4, pp. 291-302.

http://dx.doi.org/10.2307/40323822 